\title{
Development and Evaluation of a Droplet Digital PCR Assay for 8p23 $\beta$-Defensin Cluster Copy Number Determination
}

\author{
Tingting Wen ${ }^{1} \cdot$ Xianghong Zhang ${ }^{2}$ (I) $\cdot$ Christoph Lippuner $^{3} \cdot$ Marcel Schiff $^{3} \cdot$ Frank Stuber $^{3}$
}

Accepted: 4 July 2021 / Published online: 29 July 2021

(c) The Author(s), under exclusive licence to Springer Nature Switzerland AG 2021

\begin{abstract}
Introduction It has been proposed that the copy number $(\mathrm{CN})$ variation $(\mathrm{CNV})$ in $\beta$-defensin genes (DEFB) on human chromosome 8p23 determines phenotypic differences in inflammatory diseases. However, no method for accurate and easy DEFB CN quantification is yet available.

Objective Droplet digital polymerase chain reaction (ddPCR) is a novel method for CNV quantification and has been used for genes such as CCLAL, CCL3L1, AMY1, and HER2. However, to date, no ddPCR assay has been available for DEFB CN determination. In the present study, we aimed to develop and evaluate such a ddPCR assay.

Methods The assay was designed using DEFB4 and RPP30 as the target and the reference gene, respectively. To evaluate the assay, 283 DNA samples with known CNs previously determined using the multiple ligation-dependent probe amplification (MLPA) method, the current gold standard, were used as standards. To discover the optimal DNA template amount, we tested 80 to $2.5 \mathrm{ng}$ DNA by a serial of one to two dilutions of eight samples. To evaluate the reproducibility of the assay, 31 samples were repeated to calculate the intra- and inter-assay variations. To further validate the reliability of the assay, the CNs of all 283 samples were determined using ddPCR. To compare results with those using quantitative PCR (qPCR), DEFB CNs for 48 samples were determined using qPCR with the same primers and probes.

Results In a one-dimensional plot, the positive and negative droplets were clearly separated in both DEFB4 and RPP3O detection channels. In a two-dimensional plot, four populations of droplets were observed. The $20 \mathrm{ng}$ template DNA proved optimal, with either high ( $80 \mathrm{ng}$ ) or low $(10,5,2.5 \mathrm{ng})$ template input leading to ambiguous or inaccurate results. For the 31 standard samples, DEFB CNs were accurately determined with small intra- and inter-assay variations (coefficient of variation $<0.04$ for both). In the validation cohort, ddPCR provided the correct $\mathrm{CN}$ for all 283 samples with high confidence. qPCR measurements for the 48 samples produced noisy data with high uncertainty and low accuracy.
\end{abstract}

Conclusions ddPCR is an accurate, reproducible, easy-to-use, cheap, high-throughput method for DEFB CN determination. ddPCR could be applied to DEFB CN quantification in large-scale case-control studies.

\section{Introduction}

Copy number variations (CNVs) affect about $10 \%$ of the human genomic sequence $[1,2]$. Among the many genes

Xianghong Zhang

xianghong_zhang@zju.edu.cn

1 Department of Anesthesiology, The First Affiliated Hospital, Zhejiang University School of Medicine, Hangzhou,

Zhejiang, China

2 Department of Cardiac Intensive Care Unit, The Children's Hospital, Zhejiang University School of Medicine, National Clinical Research Center for Child Heath, Hangzhou, Zhejiang, China

3 Department of Anesthesiology and Pain Medicine, Bern University Hospital, Inselspital, Bern, Switzerland

\section{Key Points}

A robust method is needed to accurately measure the copy numbers of $\beta$-defensin genes on human chromosome $8 \mathrm{p} 23$, which in turn could determine susceptibility to inflammatory disease.

Digital droplet polymerase chain reaction (ddPCR) was used to measure the copy numbers, and results were compared with those obtained using multiple ligationdependent probe amplification, the current gold standard method.

ddPCR is an accurate, easy-to-use, high-throughput method that delivers reproducible copy number results. It is a good tool for large-scale case-control studies. 
with CNVs, $\beta$-defensin genes (DEFBs) located in 8p23.1 form a cluster, and the cluster exhibits extensive CNVs ranging from 2 to 12 in Caucasian populations (Fig. 1) [3, 4]. The DEFBs are a group of small cationic peptides with antimicrobial and immune regulatory properties $[5,6]$. To date, DEFB CNVs have been reported to impact on DEFB4 messenger RNA levels and probably also protein levels in many types of tissue [4, 7-10]. Based on functional analysis, genetic association studies have been conducted by different groups and DEFB CNVs found to be associated with many diseases, such as psoriasis [11,12], chronic obstructive pulmonary disease [9], Crohn's disease [7, 13], and reproductive tract infections [10]. However, most of the association studies cannot be replicated, and the discrepancy is primarily because DEFB CN determination methods are unreliable [14-17].

In our previous work, we used multiple ligation-dependent probe amplification (MLPA), a pseudogene-based paralog ratio test, and quantitative polymerase chain reaction (qPCR) to determine DEFB CN and showed that MLPA was the most reliable method among the three since it targets multiple DEFB and reference genes [16]. Therefore, MLPA is regarded as the gold standard method. However, widespread application of this method is constrained mainly by the amount of work required for experiments and data processing, its requirement for large amounts of DNA, and its high costs.

Droplet digital PCR (ddPCR) is an emerging method for $\mathrm{CN}$ determination that is gaining popularity. It is an endpoint PCR method for nucleotide acid quantification, and the PCR reactions take place in oil-wrapped nano-sized droplets. After PCR, the droplets are classified as positive or negative clusters according to the signal intensity. Thereafter, the concentration of nucleotide acid is calculated using Poisson statistics according to the percentage of positive droplets
[18-20]. Because ddPCR does not need a standard curve, it has advantages in terms of accuracy, reproducibility, and sensitivity in quantification of a wide variety of genetic materials, such as mitochondrial DNA [21], tumor-cell-free DNA [22], circulating microRNA [23], and virus copies, especially for severe acute respiratory syndrome coronavirus 2 [24], plant DNA [25], low-level somatic mosaicism [26], and even absolute gene copies in a single cell [27].

To date, ddPCR has also been tested for the determination of several gene CNVs, such as KIR [28], CCL4L [29], CCL3L1 [30], AMY1 [31], HER2 [32], and BRCA1 [33]. These genes' CNVs are mostly caused by deletion and duplication, so the CNVs range from 0 to 4 . In contrast, the DEFB CNVs have a broad dynamic range from 2 to 12 , which means it is a substantial challenge for the methodology to distinguish high CNs. Therefore, whether ddPCR can be used to accurately determine DEFB CN is unknown. The aim of the present study was to develop and evaluate a ddPCR assay for DEFB CN determination, which, with careful design and optimization, we successfully completed. In addition, we showed that ddPCR was superior to MLPA for DEFB CN determination.

\section{Materials and Methods}

\subsection{Cohort}

A cohort of 283 healthy European blood donors (Red Cross cohort, code RC) with known CNs as previously determined using MLPA was used in this study [16]. Written informed consent that covered the present study was obtained previously. This study was approved by the ethics committee of the Canton of Bern (no. KEK 041/09).
Fig. 1 Design of DEFB4 primers and probe. Assembly of the human genome region of 8p23 (GRCh38, hg38) is shown. The green arrow depicts the $\alpha$-defensin cluster. The red arrows depict two copies of the $\beta$-defensin cluster. The boxes depict genes located within the $\beta$-defensin cluster. The DEFB4 sequence was referred from GenBank with ID 1673. Yellow shading indicates the second exon of the $D E F B 4$ gene; green indicates the primer-binding sites; pink indicates the probebinding site
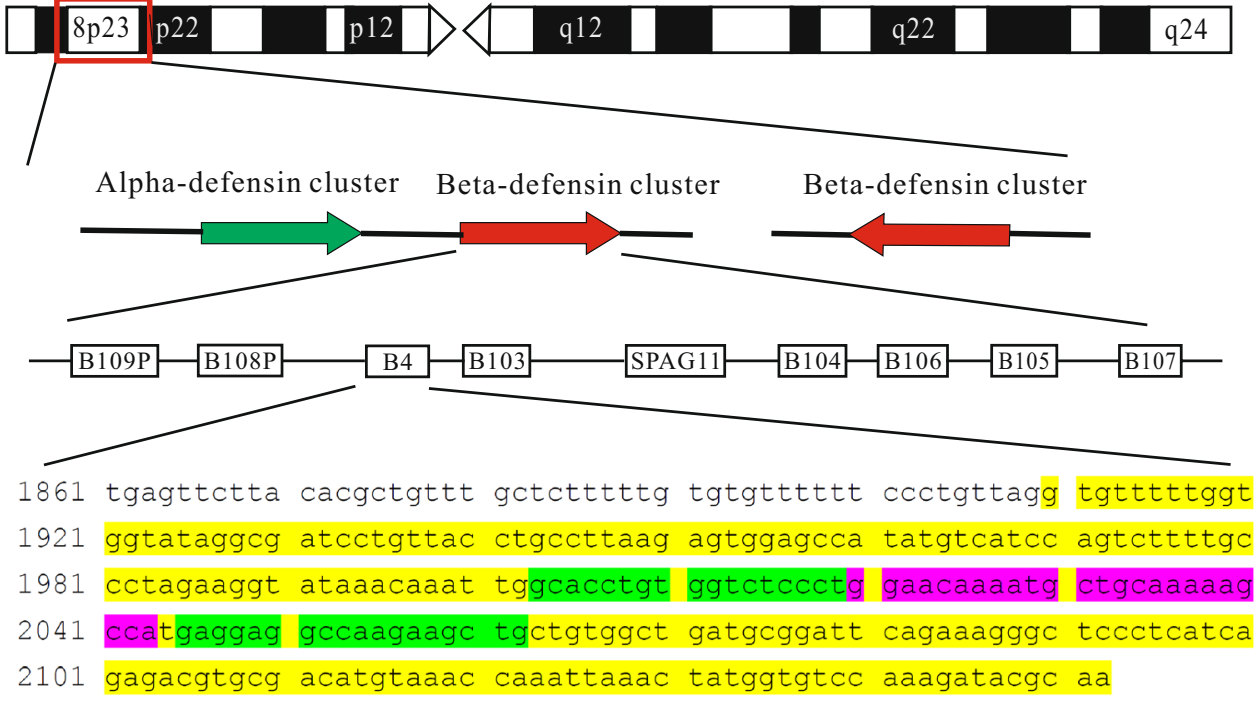


\subsection{DNA Digestion}

DNA samples were previously isolated and stored in - 20 ${ }^{\circ} \mathrm{C}$ as stock [16]. To generate a single-copy DEFB4 template, genomic DNA was digested using Msel enzyme (New England Biolabs, Switzerland) in reaction that contains approximately $100 \mathrm{ng}$ of DNA, $2.5 \mu 110 \times$ CutSmart buffer, and 2 units of Msel enzyme in a total volume of $25 \mu$ l. The digestion was performed at $37{ }^{\circ} \mathrm{C}$ for $60 \mathrm{~min}$ and then at $65^{\circ} \mathrm{C}$ for $20 \mathrm{~min}$.

\subsection{Primers and Probes}

Specific primers and probes targeting the second exon of the $D E F B 4$ gene, which is located in the DEFB cluster, were designed and generated (Fig. 1). RPP30 is a gene without CNVs in the human genome so served as a reference gene. The primers and probes for RPP 30 were commercially available and well-validated (assay ID: dHsaCP1000485, BioRad Laboratories, Hercules, CA, USA). Table 1 lists the sequences for primers and probes.

\subsection{Droplet Digital Polymerase Chain Reaction (ddPCR)}

ddPCR was performed using QX200 ${ }^{\mathrm{TM}}$ Droplet Digital PCR (Bio-Rad Laboratories) according to manufacturer's instructions. In brief, the reaction mixture $(20 \mu \mathrm{l})$ containing around 20 ng digested template DNA, $10 \mu \mathrm{lddPCR}$ supermix, 900 $\mathrm{nM}$ of each primer, and $250 \mathrm{nM}$ of each probe (Bio-Rad) was loaded into the sample well in the QX100 Droplet Generator. Then, $70 \mu \mathrm{l}$ of droplet generation oil (Bio-Rad) was loaded into the oil well. A total of $40 \mu \mathrm{l}$ of oil-water emulsion containing approximately 12,000-20,000 droplets was generated with the droplet generator and gently transferred into a separate well of a 96-well PCR plate. PCR was performed under the following thermocycling conditions: denaturation at 96 ${ }^{\circ} \mathrm{C}$ for $10 \mathrm{~min}$, amplification of 45 cycles $\left(95^{\circ} \mathrm{C}\right.$ for $5 \mathrm{~s}, 60$ ${ }^{\circ} \mathrm{C}$ for $1 \mathrm{~min}$, and $72{ }^{\circ} \mathrm{C}$ for $10 \mathrm{~s}$ ), and extension at $72{ }^{\circ} \mathrm{C}$ for $10 \mathrm{~min}$. Annealing at $60^{\circ} \mathrm{C}$ was selected after strong gradient tests and was proven optimal (data not shown). Samples with CNs from 2 to 9 as previously determined by MLPA were included in each run as positive controls. After PCR amplification, positive and negative droplets were counted using QuantaSoft software with automated clustering analysis. Nucleotide acid concentration was further calculated with Poisson statistics embedded in QuantaSoft. Raw CN values were two times the ratio of DEFB4 and RPP30 concentrations. $\mathrm{CN}$ was rounded to the nearest integer of the raw value. Samples with mid-integer raw $\mathrm{CN}$ values (those 0.30-0.70 away from an integer number) or confidence intervals wider than 1 or $<10 \%$ double-negative droplets were considered unreliable. Re-quantification was performed for
Table 1 Primers and fluorogenic probes in the digital droplet polymerase chain reaction assay

\begin{tabular}{ll}
\hline DEFB4 & \\
Forward primer & 5'-GCACCTGTGGTCTCCCT-3' \\
Reverse primer & 5'-CAGCTTCTTGGCCTCCTC-3' \\
Probe & FAM-TGGCTTTTTGCAGCATTTTGTTCC-MGB \\
RPP30 & \\
Forward primer & 5'-AGATTTGGACCTGCGAGCG-3' \\
Reverse primer & 5'-GAGCGGCTGTCTCCACAAGT-3' \\
Probe & HEX-TTCTGACCTGAAGGCTCTGCGCG-BHQ1
\end{tabular}

$D E F B \beta$-defensin genes

samples from DNA digestion. Two independent runs were conducted for each sample, and $\mathrm{CN}$ was determined only when concordant results were obtained from two runs.

\subsection{Real-Time Quantitative PCR (qPCR)}

Using the same primers and probes as for ddPCR, qPCR was carried out in the same reaction mixture using Roche LightCycler 480 thermocycler under the same reaction conditions as for ddPCR. DEFB CN was inferred using the $\Delta \Delta \mathrm{Cp}$ method using samples with $\mathrm{CNs}$ of 2 as calibrator. Independent replicate runs were also performed for each sample.

\subsection{Statistical Analysis}

Statistical analyses were conducted in Excel, and plotting was performed using GraphPad Prism 7.0 for Windows (GraphPad Software, San Diego, CA, USA). The coefficient of variation (CV) for intra- and inter-assay variation analysis was calculated using means \pm standard deviations.

\section{Results}

\subsection{Performance of ddPCR Assay for $\beta$-Defensin Genes (DEFB) Copy Number Determination}

In a successful measurement, the positive and negative droplets were clearly separated in both DEFB4 and RPP30 detection channels (Fig. 2a-d). Typically, four populations of droplets in a two-dimensional plot were observed in this duplex ddPCR assay (Fig. 2e). DEFB4 and RPP30 concentrations were calculated to infer DEFB CN (Fig. 3a). Samples with CNs from 2 to 9 as previously determined with MLPA were used as a preliminary test of the reliability of the ddPCR assay. All eight samples showed concordant CNs (Fig. 3b). 


\subsection{Optimization of DNA Template Amount for ddPCR}

The DNA template amount is critical for the calculation of absolute nucleic acid concentration in a ddPCR assay [34, 35]. To find out the suitable DNA template amount, DNA input were tested from 80 to $2.5 \mathrm{ng}$ by a serial of one to two dilutions of eight samples with CNs from 2 to 9 . PCR reaction with $20 \mathrm{ng}$ input DNA was found to be sufficient to obtain the best result (although $40 \mathrm{ng}$ DNA template also provided correct $\mathrm{CN}$ results). From our experiment, either high (80 ng) or low (10, 5, and 2.5 ng) amounts of DNA template led to ambiguous (values between 0.3 and 0.7 around the integer number) or incorrect CNs (Fig. 4). High template input ( $80 \mathrm{ng}$ ) resulted in too few negative events for DEFB4 and/or RPP30 genes (Fig. 1a in the electronic supplementary material [ESM]), especially for high $\mathrm{CN}$ samples. In contrast, low template input ( $\leq 10 \mathrm{ng}$ ) led to ambiguous or discordant $\mathrm{CNs}$ for both low and high $\mathrm{CN}$ samples because there were too few positive events for target and/or reference genes (Fig. 1b in the ESM).

\subsection{Evaluation of Assay Reproducibility}

To evaluate the reproducibility of the ddPCR assay, $31 \mathrm{RC}$ samples with CNs from 2 to 9 were tested in triplicate in the same run and in singleplex assay in three independent runs for intra-assay and inter-assay variation, respectively. Concordant integer CNs were obtained among inter- and intra-assay repeats. Moreover, $\mathrm{CV}$ s for both intra- and interassay were $<0.04$ (Table 2).

\subsection{Validation of the ddPCR Assay in a European Population}

To further validate the reliability of the ddPCR assay in a random population, DEFB CNs of all 283 samples from healthy blood donors were determined by ddPCR. To increase the reliability of the data, independent duplicates were run for each sample, and concordance between duplicates was regarded as successful determination. Eventually, concordant integer $\mathrm{CNs}$ between duplicates were obtained for all samples, indicating that the call rate for CNs was $100 \%$. In addition, CNs determined by ddPCR were completely identical to those determined using MLPA (see Table 1 in the ESM). The excellent congruence of the two methods was also shown in Bland-Altman plots as the ratio was always equal to one (Fig. 5). The raw data for $\mathrm{CNs}$ clustered around the integer number and clear intervals between neighboring $\mathrm{CNs}$ were shown in a scatter plot as gaps (Fig. 6). Figure 7 shows the frequencies of the $\mathrm{CN}$ distribution patterns.

\subsection{Quantification of DEFB CN by qPCR}

Using the same primers, probes, and working conditions, we conducted qPCR with 48 samples. Inconsistent CNs between replicates were observed for 24 samples (50\%). For eight samples (16.7\%), the CNs were underestimated compared with ddPCR, although consistent results were obtained from replicates. Moreover, qPCR tended to underestimate $\mathrm{CNs}$, especially for samples with more than four CNs $(54.1 \%)$; undercounting by two copies was also observed. Only 15 samples (31.3\%) with consistent CNs between replicates showed concordant results with ddPCR (see Table 2 in the ESM).

$\mathrm{b}$

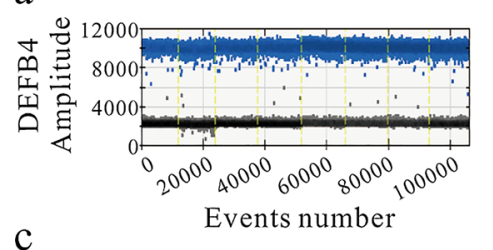

C

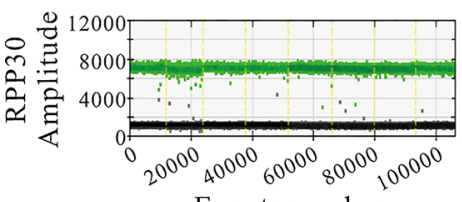

Events number

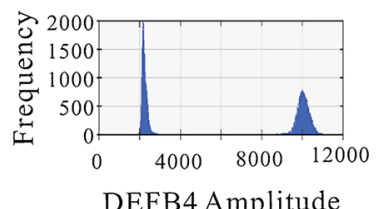

d

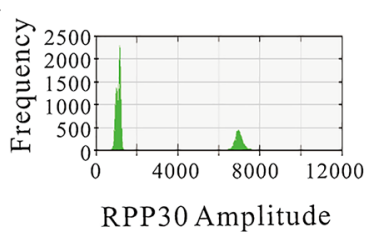

e

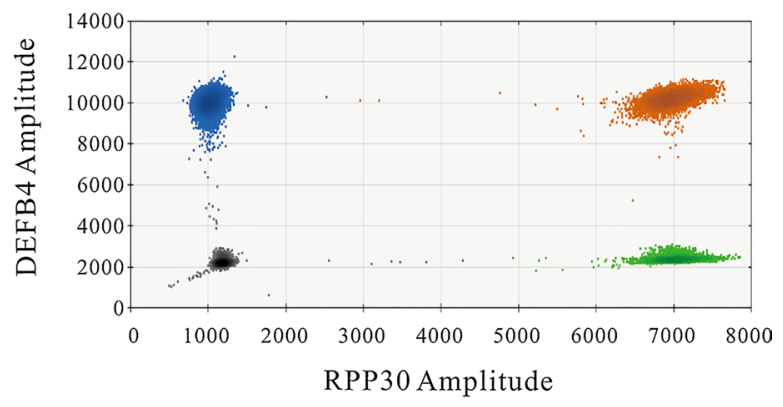

Fig. 2 Droplets plot of the digital droplet polymerase chain reaction assay. Eight samples with copy numbers from 2 to 9 previously determined using multiple ligation-dependent probe amplification were used. a-d One-dimensional amplitude plot. a $D E F B 4$ positive and negative droplets are presented as blue dots and gray dots, respectively; b the corresponding histogram. c RPP 30 positive and negative droplets are presented as green dots and gray dots, respectively; d the corresponding histogram. e Two-dimension amplitude plot. Gray shading indicates double negative; blue shading indicates FAM-positive; green shading indicates HEX-positive; orange shading indicates double positive 
Fig. 3 Quantification results of the digital droplet polymerase chain reaction (ddPCR) assay. The same eight samples with copy numbers (CNs) from 2 to 9 were used. a Concentrations of DEFB4 (blue circles) and $R P P 30$ (green circles). The left vertical axis indicates $D E F B 4$ concentration. The right vertical axis indicates $R P P 30$ concentration. The absolute concentrations are labeled beside the circles. The error bars represent the $95 \%$ Poisson confidence interval. b CN determined by ddPCR. The error bars represent the $95 \%$ confidence interval of the calculated raw $\mathrm{CN}$ a

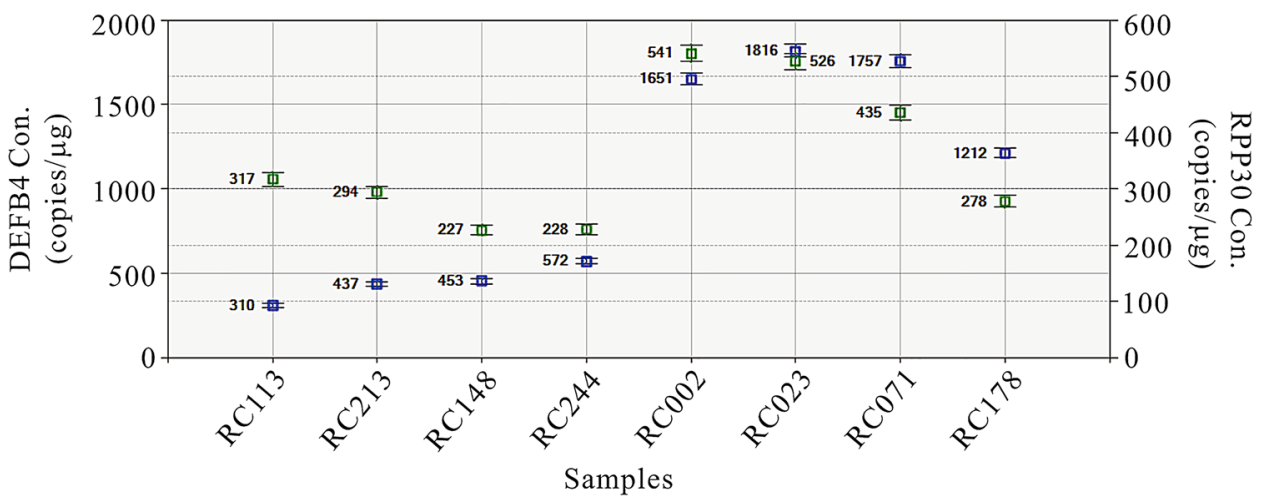

b

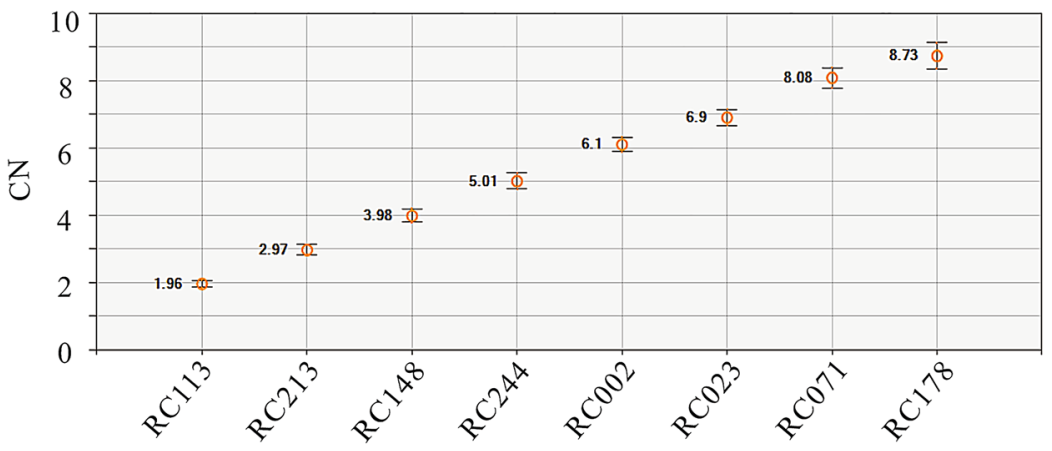

Samples

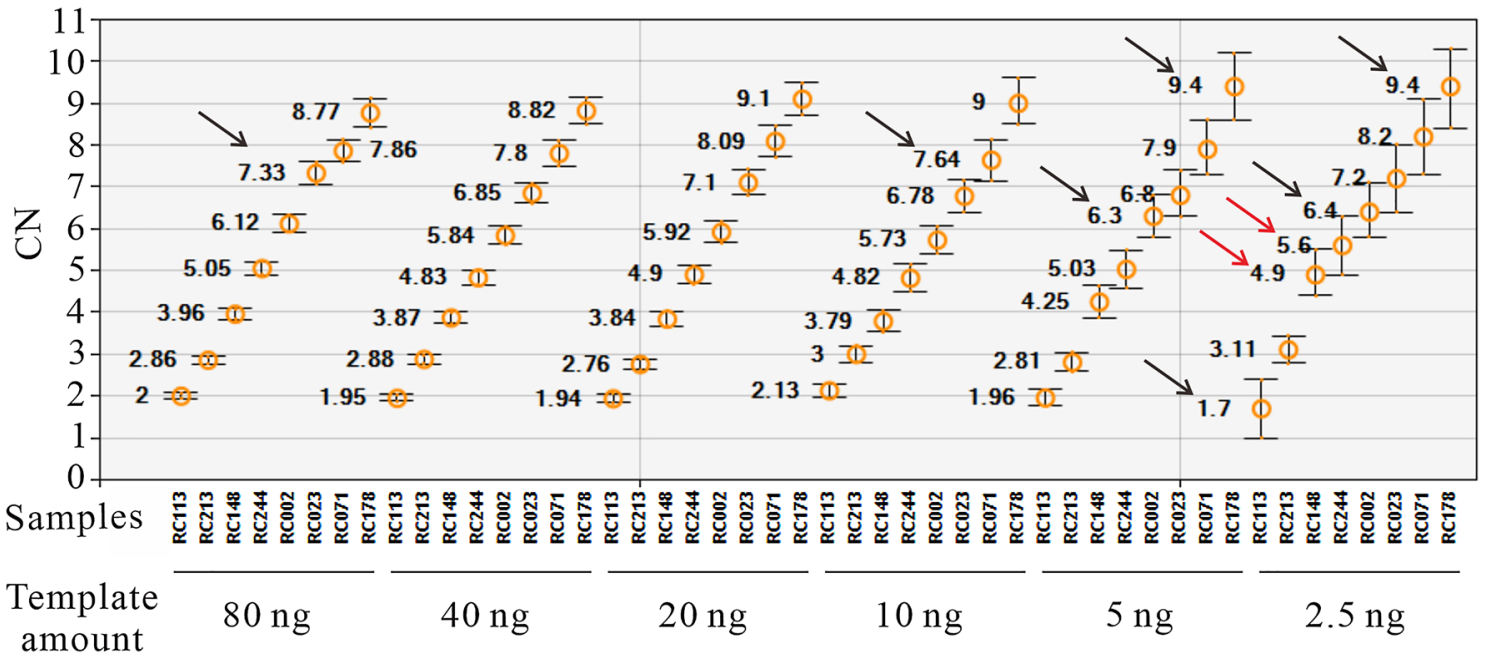

Fig. 4 Effect of template amount on $\beta$-defensin genes (DEFB) copy number $(\mathrm{CN})$ determination in the digital droplet polymerase chain reaction (ddPCR) assay. The same eight samples with CNs from 2 to 9 and template amounts of $80,40,20,10,5$, and 2.5 ng were tested.

\section{Discussion}

In the present study, we developed a new assay for DEFB $\mathrm{CN}$ determination using ddPCR. We also found that, with a call rate of $100 \%$, ddPCR could accurately and
Yellow circles represent inferred CN; bars indicate the 95\% confidence interval of the raw CNs. Black arrows indicate ambiguous $\mathrm{CN}$ values; red arrows indicate inconsistent $\mathrm{CN}$ values

reproducibly determine DEFB CNs. Validation of ddPCR in 283 samples of healthy blood donors showed integer CNs consistent with those obtained with MLPA.

The reliability and high reproducibility of our ddPCR assay for DEFB CN determination could be attributed 
Table 2 Intra- and inter-assay variation

\begin{tabular}{|c|c|c|c|c|c|c|}
\hline \multirow[t]{2}{*}{$\mathrm{CN}$} & \multicolumn{3}{|c|}{ Intra-assay variation } & \multicolumn{3}{|c|}{ Inter-assay variation } \\
\hline & Mean & SD & $\mathrm{CV}$ & Mean & SD & $\mathrm{CV}$ \\
\hline 2 & 1.99 & 0.05 & 0.03 & 1.97 & 0.06 & 0.03 \\
\hline 3 & 2.99 & 0.07 & 0.03 & 3.01 & 0.06 & 0.02 \\
\hline 4 & 3.93 & 0.08 & 0.02 & 3.94 & 0.08 & 0.02 \\
\hline 5 & 4.94 & 0.08 & 0.02 & 5.01 & 0.09 & 0.02 \\
\hline 6 & 5.94 & 0.15 & 0.02 & 5.93 & 0.13 & 0.02 \\
\hline 7 & 6.90 & 0.15 & 0.02 & 6.97 & 0.15 & 0.02 \\
\hline 8 & 7.87 & 0.10 & 0.01 & 7.93 & 0.11 & 0.01 \\
\hline 9 & 9.00 & 0.14 & 0.02 & 9.13 & 0.20 & 0.02 \\
\hline
\end{tabular}

$C N$ copy number, $C V$ coefficient of variation, $S D$ standard deviation

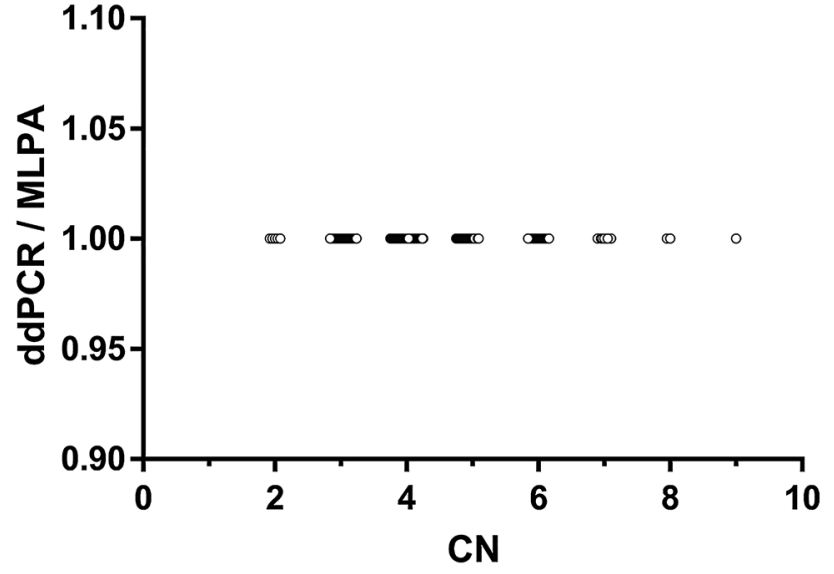

Fig. 5 Bland-Altman plot for comparison between digital droplet polymerase chain reaction (ddPCR) and multiple ligation-dependent probe amplification (MLPA). The plots show the ratio of the two methods against their mean. The dots were offset to allow individual measurements to be distinguished

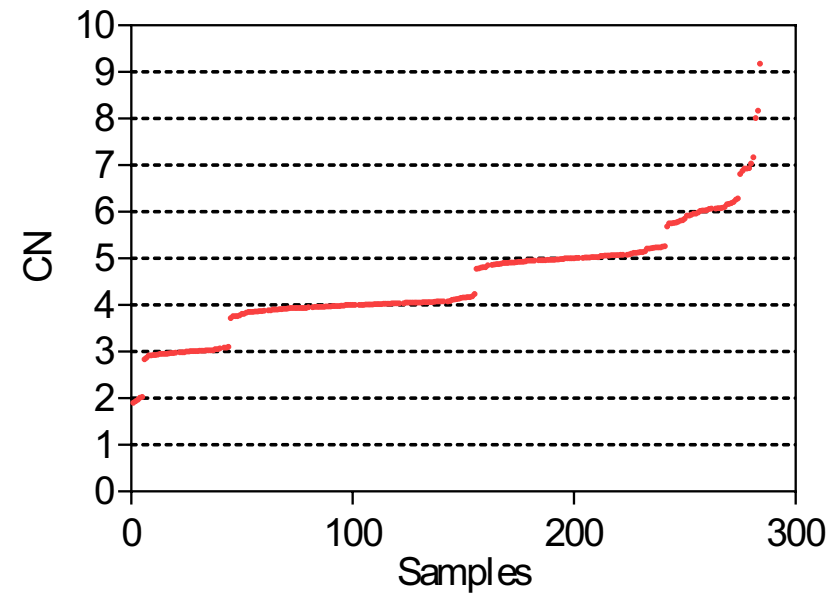

Fig. 6 Scatter plot of raw copy number $(\mathrm{CN})$ values of 283 samples determined by digital droplet polymerase chain reaction. Raw $\mathrm{CN}$ values were plotted in ascending order

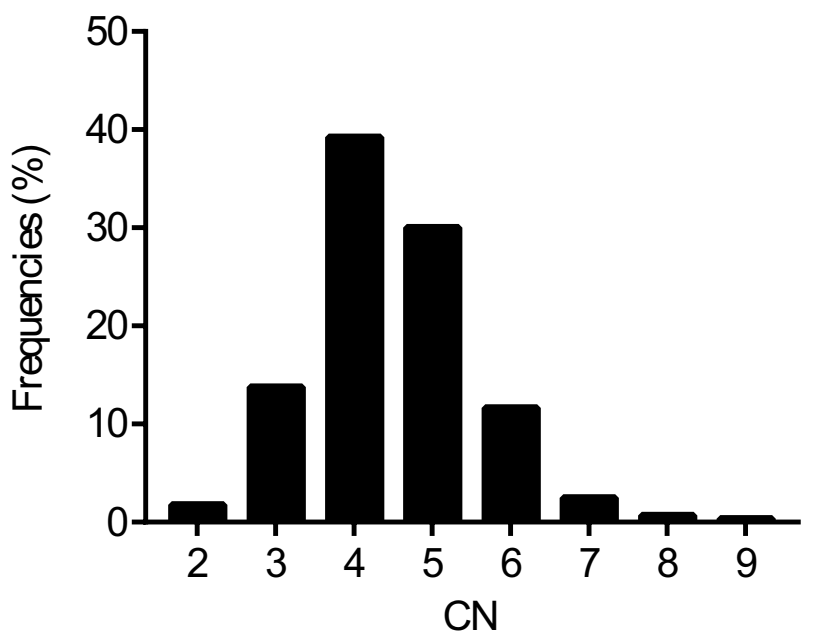

Fig. $7 \beta$-Defensin genes (DEFB) copy number $(\mathrm{CN})$ distribution in 283 individuals of European ancestry. DEFB CNs were determined by digital droplet polymerase chain reaction

to the following. First and most important, ddPCR is an endpoint but not real-time quantification method. After PCR amplification, droplets are assigned as either positive or negative in a binomial way, and nucleotide acid concentration is further calculated using Poisson statistics. For positive droplets, defined as fluorescent signals above the threshold, no perfect amplification efficiency is needed [19, 20, 36]. This feature makes ddPCR much more tolerant to inhibitory factors, which can decrease the amplification efficiency [37-39]. Second, the primers and probes were designed to target the second exon of the $D E F B 4$ gene, which is a sequence conservative region. It has been reported that perfect binding of the primer and probes to template is essential to reduce the intermediate droplets between the positive and the negative (also called rain) $[40,41]$. As shown in our results, a clear separation between positive and negative clusters (little or even 
no rain) ensured accurate $\mathrm{CN}$ inference. It is reasonable that single nucleotide variations (SNVs) in primer- or/and probe-binding sites of the target gene may lead to unsuccessful amplification or/and indication of some droplets. Consequently, they can cause underestimation of nucleotide acid concentration for the target gene and eventually undercount the gene CNs. For this reason, primer- and probe-binding sites must be checked using tools such as NCBI Variation Viewer to avoid SNVs before starting the assay. Third, we used the optimal DNA template amount in the ddPCR assay. The theoretical and calculation fundament of ddPCR also has limitations when the percentage of positive or negative droplets are at their extremes [18]. As tested in our ddPCR assay, 80 ng DNA input showed an extremely high percentage of positive droplets for the $D E F B 4$ gene and caused ambiguous gene $\mathrm{CN}$ determination, especially for high $\mathrm{CN}$ samples (nucleic acid concentration of target gene increases fold-wise after enzyme digestion) (Fig. 4; Fig. 1a in the ESM). Meanwhile, Härmälä et al. [42] also found that a high DNA template amount could lead to ambiguous $\mathrm{CN}$ determinations using ddPCR for the haptoglobin gene. We think that too high nucleic acid concentrations of the target gene could lead to nearly saturated positive target droplets and consequently affect $\mathrm{CN}$ calculation. Conversely, low template amounts $(10,5$, and $2.5 \mathrm{ng})$ led to a too low proportion of positive droplets for DEFB4 and/or RPP30 gene(s) and also affected the correct interpretation of target and/or reference gene(s) concentrations (Fig. 1b in the ESM). Accordingly, the CNs cannot be calculated correctly (Fig. 4). A wide range of template amounts, from 7 to $80 \mathrm{ng}$ was reported for determining different gene CNs [29, 31, 41, 42]. Considering our results (20 ng), we speculate that the optimal DNA template amount is gene and assay specific. Therefore, we suggest that the template amount be tested before applying a new ddPCR assay for $\mathrm{CN}$ determination.

ddPCR has advantages over MLPA for DEFB CN determination. As reported in our previous work, compared with the paralog ratio test and qPCR, MLPA is the most reliable and accurate method for DEFB CN determination $[16,17]$. Here, we showed that results obtained with ddPCR are as accurate and reproducible as those with MLPA. The intraand inter- assay variations of ddPCR were low, as indicated by small CVs $(<0.04)$, which is comparable to that with MLPA in our previous study [17]. However, ddPCR is easier to establish and less time- and labor-intensive because fewer experimental procedures and calculation steps are needed. For this reason, it may be widely applied for large population screening. In addition, a lower template amount is needed for ddPCR. This feature is important in clinical settings with limited experimental material. Moreover, ddPCR is more tolerant to low-quality (and even degraded) DNA templates
Table 3 Comparison between digital droplet polymerase chain reaction and multiple ligation-dependent probe amplification

\begin{tabular}{llc}
\hline Attribute & ddPCR & MLPA \\
\hline Accurate & $\sqrt{ }$ & $\sqrt{ }$ \\
Reproducible & $\sqrt{ }$ & $\sqrt{ }$ \\
Easy to establish & $\sqrt{ }$ & \\
Easy to infer CN & $\sqrt{ }$ & \\
Time saving & $\sqrt{ }$ & \\
Labor saving & $\sqrt{ }$ & \\
Small DNA template & $\sqrt{ }$ & \\
Tolerant of DNA quality & $\sqrt{ }$ & \\
Cheap & $\sqrt{ }$ & \\
\hline
\end{tabular}

$C N$ copy number, $d d P C R$ digital droplet polymerase chain reaction, $M L P A$ multiple ligation-dependent probe amplification

because the amplicons are short and the PCR efficiency is not as important. In contrast, MLPA is quite sensitive to the quality of the DNA template, which could affect hybridization and subsequent amplification. Lastly, ddPCR is less expensive to use than MLPA (at \$US1 vs. \$US20 per assay, respectively). Table 3 summarizes a comparison of ddPCR and MLPA.

ddPCR is even more advantageous over qPCR for DEFB $\mathrm{CN}$ determination. In the present study, we showed that qPCR could underestimate DEFB CNs and that $\mathrm{CN}$ results were unreproducible even when we used the same reagents and reaction conditions as in ddPCR. The major difference in experimental procedures is the generation of microdroplets in ddPCR, through which PCR reaction is divided into thousands of parts and PCR is performed in independent compartments. In this manner, ddPCR may suffer from less competition for materials, including templates, primers, probes, and polymerase. In contrast, this competition cannot be avoided in qPCR so could cause dropout of some copies, especially in high $\mathrm{CN}$ samples. The other major difference is the calculation algorithm for $\mathrm{CN}$ in which PCR efficiency is less dependent for ddPCR. However, qPCR depends on PCR efficiency and cycle threshold values to infer template concentrations. Thus, qPCR is more sensitive to PCR inhibitors.

\section{Conclusions}

This study showed that ddPCR is an accurate, reproducible, easy-to-use, cheap, high-throughput method for DEFB CN determination. ddPCR could be applied to DEFB CN quantification in large-scale case-control studies.

Supplementary Information The online version contains supplementary material available at https://doi.org/10.1007/s40291-021-00546-2. 
Acknowledgements The authors thank Prof. Dr. Rolf Jaggi (DKF Genomics Core Facility, University of Bern, Switzerland) for offering access to the ddPCR instruments. We also thank Dr. Gertrud Heimbeck (Bio-Rad Laboratories AG, Reinach, Switzerland) for assistance in the design of the ddPCR assay.

\section{Declarations}

Funding This work was supported by a grant to $\mathrm{XZ}$ from "Wissenschaftlichen Fonds der Universitätsklinik für Anästhesiologie und Schmerztherapie Bern" (ZHXF-1-14).

Conflict of interest Tingting Wen, Xianghong Zhang, Christoph Lippuner, Marcel Schiff, and Frank Stuber have no conflicts of interest that are directly relevant to the content of this article.

Ethics approval All procedures performed in studies involving human participants were in accordance with the ethical standards of the institutional and/or national research committee and with the 1964 Helsinki declaration and its later amendments or comparable ethical standards. This study was approved by the Ethics Committee of the Canton of Bern (No. KEK 041/09).

Consent to participate Informed consent was obtained from all individual participants included in the study.

Consent for publication Participants signed informed consent regarding publishing their data.

Availability of data and material All data generated or analyzed during this study are included in this published article and its supplementary information files.

\section{Code availability Not applicable}

Author contributions FS initiated and coordinated the study. XZ designed the study. WT, MS, CL and XZ performed the experiments. WT and XZ analyzed the data and drafted the manuscript. CL and FS revised the manuscript. All authors read and approved the final manuscript.

\section{References}

1. Redon R, Ishikawa S, Fitch KR, Feuk L, Perry GH, Andrews TD, et al. Global variation in copy number in the human genome. Nature. 2006;444(7118):444-54.

2. Conrad DF, Pinto D, Redon R, Feuk L, Gokcumen O, Zhang Y, et al. Origins and functional impact of copy number variation in the human genome. Nature. 2010;464(7289):704-12.

3. Hollox EJ, Armour JA, Barber JC. Extensive normal copy number variation of a beta-defensin antimicrobial-gene cluster. Am J Hum Genet. 2003;73(3):591-600.

4. Groth M, Szafranski K, Taudien S, Huse K, Mueller O, Rosenstiel $\mathrm{P}$, et al. High-resolution mapping of the 8p23.1 beta-defensin cluster reveals strictly concordant copy number variation of all genes. Hum Mutat. 2008;29(10):1247-54.

5. Ganz T. Defensins: antimicrobial peptides of innate immunity. Nat Rev Immunol. 2003;3(9):710-20.

6. Semple F, Dorin JR. beta-Defensins: multifunctional modulators of infection, inflammation and more? J Innate Immun. 2012;4(4):337-48.
7. Fellermann K, Stange DE, Schaeffeler E, Schmalzl H, Wehkamp $\mathrm{J}$, Bevins CL, et al. A chromosome 8 gene-cluster polymorphism with low human beta-defensin 2 gene copy number predisposes to Crohn disease of the colon. Am J Hum Genet. 2006;79(3):439-48.

8. Groth M, Wiegand C, Szafranski K, Huse K, Kramer M, Rosenstiel $\mathrm{P}$, et al. Both copy number and sequence variations affect expression of human DEFB4. Genes Immun. 2010;11(6):458-66.

9. Janssens W, Nuytten H, Dupont LJ, Van Eldere J, Vermeire S, Lambrechts D, et al. Genomic copy number determines functional expression of $\{$ beta $\}$-defensin 2 in airway epithelial cells and associates with chronic obstructive pulmonary disease. Am J Respir Crit Care Med. 2010;182(2):163-9.

10. James CP, Bajaj-Elliott M, Abujaber R, Forya F, Klein N, David AL, et al. Human beta defensin (HBD) gene copy number affects HBD2 protein levels: impact on cervical bactericidal immunity in pregnancy. Eur J Hum Genet. 2018;26(3):434-9.

11. Hollox EJ, Huffmeier U, Zeeuwen PL, Palla R, Lascorz J, RodijkOlthuis $\mathrm{D}$, et al. Psoriasis is associated with increased beta-defensin genomic copy number. Nat Genet. 2008;40(1):23-5.

12. Stuart PE, Huffmeier U, Nair RP, Palla R, Tejasvi T, Schalkwijk $\mathrm{J}$, et al. Association of beta-defensin copy number and psoriasis in three cohorts of European origin. J Invest Dermatol. 2012;132(10):2407-13.

13. Bentley RW, Pearson J, Gearry RB, Barclay ML, McKinney C, Merriman TR, et al. Association of higher DEFB4 genomic copy number with Crohn's disease. Am J Gastroenterol. 2010;105(2):354-9.

14. Aldhous MC, Abu Bakar S, Prescott NJ, Palla R, Soo K, Mansfield $\mathrm{JC}$, et al. Measurement methods and accuracy in copy number variation: failure to replicate associations of beta-defensin copy number with Crohn's disease. Hum Mol Genet. 2010;19(24):4930-8.

15. Wain LV, Odenthal-Hesse L, Abujaber R, Sayers I, Beardsmore $\mathrm{C}$, Gaillard EA, et al. Copy number variation of the beta-defensin genes in europeans: no supporting evidence for association with lung function, chronic obstructive pulmonary disease or asthma. PloS One. 2014;9(1):e84192.

16. Zhang X, Muller S, Moller M, Huse K, Taudien S, Book M, et al. 8 p23 beta-defensin copy number determination by single-locus pseudogene-based paralog ratio tests risk bias due to low-frequency sequence variations. BMC Genom. 2014;15:64.

17. Perne A, Zhang X, Lehmann L, Groth M, Stuber F, Book M. Comparison of multiplex ligation-dependent probe amplification and real-time PCR accuracy for gene copy number quantification using the beta-defensin locus. Biotechniques. 2009;47(6):1023-8.

18. Quan PL, Sauzade M, Brouzes E. dPCR: a technology review. sensors (Basel, Switzerland). 2018;18(4):1271.

19. Hindson BJ, Ness KD, Masquelier DA, Belgrader P, Heredia NJ, Makarewicz AJ, et al. High-throughput droplet digital PCR system for absolute quantitation of DNA copy number. Anal Chem. 2011;83(22):8604-10.

20. Pinheiro LB, Coleman VA, Hindson CM, Herrmann J, Hindson $\mathrm{BJ}$, Bhat $\mathrm{S}$, et al. Evaluation of a droplet digital polymerase chain reaction format for DNA copy number quantification. Anal Chem. 2012;84(2):1003-11.

21. O'Hara R, Tedone E, Ludlow A, Huang E, Arosio B, Mari D, et al. Quantitative mitochondrial DNA copy number determination using droplet digital PCR with single-cell resolution. Genome Res. 2019;29(11):1878-88.

22. Zivanovic Bujak A, Weng CF, Silva MJ, Yeung M, Lo L, Ftouni $\mathrm{S}$, et al. Circulating tumour DNA in metastatic breast cancer to guide clinical trial enrolment and precision oncology: a cohort study. PLoS Med. 2020;17(10):e1003363.

23. Zhang Y, Wang Y, Zhang L, Xia L, Zheng M, Zeng Z, et al. Reduced platelet miR-223 induction in Kawasaki disease leads to severe coronary artery pathology through a miR-223/PDGFR $\beta$ vascular smooth muscle cell axis. Circ Res. 2020;127(7):855-73. 
24. Yu F, Yan L, Wang N, Yang S, Wang L, Tang Y, et al. Quantitative detection and viral load analysis of SARS-CoV-2 in infected patients. Clin Infect Dis. 2020;71(15):793-8.

25. Wu Z, Waneka G, Broz AK, King CR, Sloan DB. MSH1 is required for maintenance of the low mutation rates in plant mitochondrial and plastid genomes. Proc Natl Acad Sci USA. 2020;117(28):16448-55.

26. Karolak JA, Liu Q, Xie NG, Wu LR, Rocha G, Fernandes S, et al. Highly sensitive blocker displacement amplification and droplet digital PCR reveal low-level parental FOXF1 somatic mosaicism in families with alveolar capillary dysplasia with misalignment of pulmonary veins. J Mol Diagn. 2020;22(4):447-56.

27. Santeramo I, Bagnati M, Harvey EJ, Hassan E, Surmacz-Cordle B, Marshall D, et al. Vector copy distribution at a single-cell level enhances analytical characterization of gene-modified cell therapies. Mol Ther Methods Clin Dev. 2020;12(17):944-56.

28. Roberts CH, Jiang W, Jayaraman J, Trowsdale J, Holland MJ, Traherne JA. Killer-cell Immunoglobulin-like receptor gene linkage and copy number variation analysis by droplet digital PCR. Genome Med. 2014;6(3):20.

29. Bharuthram A, Paximadis M, Picton AC, Tiemessen CT. Comparison of a quantitative real-time PCR assay and droplet digital PCR for copy number analysis of the CCL4L genes. Infect Genet Evol. 2014;25:28-35.

30. Ben Kilani MS, Achour Y, Perea J, Cornelis F, Bardin T, Chaudru $\mathrm{V}$, et al. Characterization of copy number variants for CCL3L1 gene in rheumatoid arthritis for French trio families and Tunisian cases and controls. Clin Rheumatol. 2016;35(8):1917-22.

31. Ooi DS, Tan VM, Ong SG, Chan YH, Heng CK, Lee YS. Differences in AMY1 gene copy numbers derived from blood, buccal cells and saliva using quantitative and droplet digital PCR methods: flagging the pitfall. PLoS ONE. 2017;12(1):e0170767.

32. Yu R, Xuan W, Zhou L, Luo Y, Liu X, Xiong P, et al. Detection of HER2 amplification in formalin-fixed paraffin-embedded breast carcinoma tissue with digital PCR using two TFF3 sequences as internal reference. Exp Mol Pathol. 2018;104(3):235-8.
33. Oscorbin I, Kechin A, Boyarskikh U, Filipenko M. Multiplex ddPCR assay for screening copy number variations in BRCA1 gene. Breast Cancer Res Treat. 2019;178(3):545-55.

34. Strain MC, Lada SM, Luong T, Rought SE, Gianella S, Terry VH, et al. Highly precise measurement of HIV DNA by droplet digital PCR. PLoS ONE. 2013;8(4):e55943.

35. Zhang Y, Xu Y, Zhong W, Zhao J, Chen M, Zhang L, et al. Total DNA input is a crucial determinant of the sensitivity of plasma cell-free DNA EGFR mutation detection using droplet digital PCR. Oncotarget. 2017;8(4):5861-73.

36. Hindson CM, Chevillet JR, Briggs HA, Gallichotte EN, Ruf IK, Hindson BJ, et al. Absolute quantification by droplet digital PCR versus analog real-time PCR. Nat Methods. 2013;10(10):1003-5.

37. Nixon G, Garson JA, Grant P, Nastouli E, Foy CA, Huggett JF. Comparative study of sensitivity, linearity, and resistance to inhibition of digital and nondigital polymerase chain reaction and loop mediated isothermal amplification assays for quantification of human cytomegalovirus. Anal Chem. 2014;86(9):4387-94.

38. Dingle TC, Sedlak RH, Cook L, Jerome KR. Tolerance of droplet-digital PCR vs real-time quantitative PCR to inhibitory substances. Clin Chem. 2013;59(11):1670-2.

39. Zhao Y, Xia Q, Yin Y, Wang Z. Comparison of droplet digital PCR and quantitative PCR assays for quantitative detection of Xanthomonas citri subsp. citri. PLoS ONE. 2016;11(7):e0159004.

40. Hughesman CB, Lu XJ, Liu KY, Zhu Y, Poh CF, Haynes C. A Robust protocol for using multiplexed droplet digital PCR to quantify somatic copy number alterations in clinical tissue specimens. PLoS ONE. 2016;11(8):e0161274.

41. Bell AD, Usher CL, McCarroll SA. Analyzing copy number variation with droplet digital PCR. Methods Mol Biol. 2018;1768:143-60.

42. Härmälä SK, Butcher R, Roberts $\mathrm{CH}$. Copy number variation analysis by droplet digital PCR. Methods Mol Biol. 2017;1654:135-49. 